\title{
Téoros
}

Revue de recherche en tourisme

\section{Le tourisme d'aventure. Pour quelle aventure ?}

\section{Jean-Claude Jay-Rayon et Brigitte Morneau}

Volume 13, numéro 3, automne 1994

Le tourisme d'aventure : vers la maturité ?

URI : https://id.erudit.org/iderudit/1077108ar

DOI : https://doi.org/10.7202/1077108ar

Aller au sommaire du numéro

Éditeur(s)

Université du Québec à Montréal

ISSN

0712-8657 (imprimé)

1923-2705 (numérique)

Découvrir la revue

Citer cet article

Jay-Rayon, J.-C. \& Morneau, B. (1994). Le tourisme d'aventure. Pour quelle aventure ? Téoros, 13(3), 11-16. https://doi.org/10.7202/1077108ar d'utilisation que vous pouvez consulter en ligne.

https://apropos.erudit.org/fr/usagers/politique-dutilisation/ 


\section{Le tourisme d'aventure. Pour quelle aventure?}

\section{Jean-Claude Jay-Rayon, en collaboration avec Brigitte Morneau*}

\section{Appliquer l'histoire... à l'aventure}

Le tourisme d'aventure - ou du moins ce que l'on place globalement sous ce vocable aujourd'hui - possède sa propre base historique et des origines culturelles diverses qui remontent principalement au $\mathrm{XVII}^{e}, \mathrm{XVIII}^{\mathrm{e}}$, et surtout au $\mathrm{XIX}^{\mathrm{e}}$ siècle. Celui des grands voyages, expéditions et explorations pré-modernes de l'Océanie, de l'Afrique, de l'Asie...

Toutefois, en ce qui a trait aux Amériques, à l'Australie, à la Nouvelle-Zélande et à certains endroits de l'Asie, nous pourrions en resituer le cadre dans la suite des événements successifs de la période de colonisation, de découverte et de conquîte des terres et océans par l'Europe.

Il est important de bien saisir les fondements géo-culturels du tourisme d'aventure car, sans toujours trops'en apercevoir, certainesorganisations puisentparfois avec plus ou moins d'adresse, aux sources du passé, ce qui porte atteinte à la qualité du produit aventure et pourrait le rendre à la longue, désuet.

En ce sens, si le tourisme d'aventure est envisagé professionnellement-au-delà des simples techniques et activités - il apparaît basé sur un ensemble référentiel intéressant mais, souvent négligél Par exemple, on se rend compte que l'aventure s'appuie sur une vision mythique des territoires et de leurs habitants - une vulgarisation ethnologique concernant les populations autochtones et aborigènes - des images scolaires, apprises dès le jeune âge - ainsi qu'un imaginaire relié à l'espace grandiose et atemporel (i).

En fait, le tourisme d'aventure repose sur un ensemble conceptuel composé de notions diverses et complexes dépendant des cultures auxquelles il s'adresse en terme

Monsieur Jean-Claude Jay-Payon est concepteurchercheur et maitre de conithrence. Madame Brigitte Morneau est associee experte au sein de la firme Tangram et se spécialise dans la mise en valeur touristique d'environnements naturels et culturels.

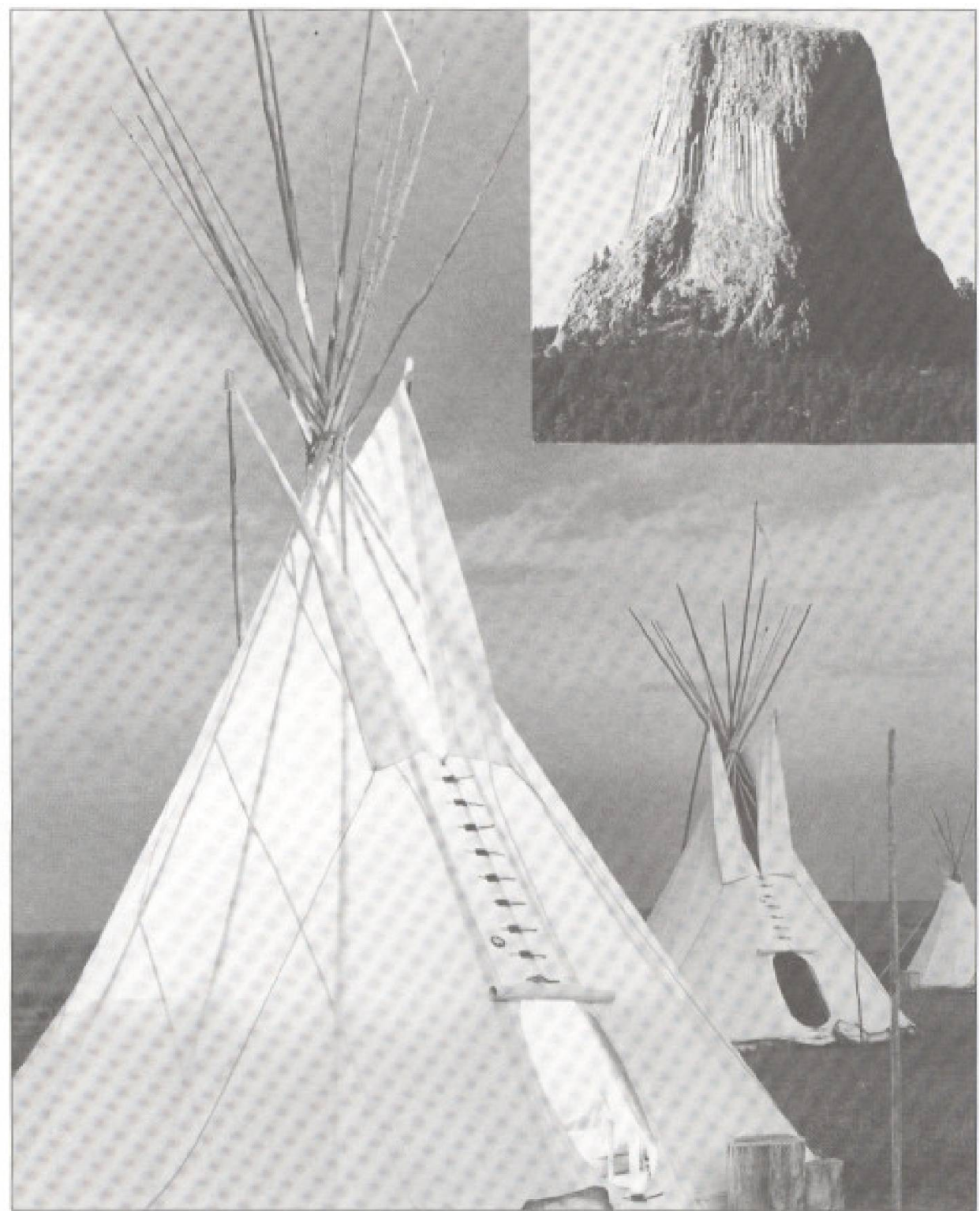

Les atepeew. Village amérindien reconstitué dans le grand-espace des plaines du Wyoming, lâ où se réunissaient les Sioux, il y a plus de 100 ans. A environ 90 miles du site sacré de Mateo Tepee. Les terrains de la nowvelle gendration-aventure de l'Ouest. Photographo: Kent Barker

d'offre. Il fait appel indirectement aussi bien à la conquête militaireet à la résistance légendaire du soldat dans un grand espace hostile, d'où cette approche de défi et de combat contre la nature - quà la longue route de la soie vers l'Orient, à la rencontre des peuples civilisés - qu'à la découverte du monde par les grands explorateurs: Cook, Darwin...
Àcelas'ajoutent-de façon plus récente-les observationsscientifiques dela fin du XIX, début $\mathrm{XX}^{t}$ siècle; celles des glaciers, gouffres, volcans, abisses, montagnes, déserts, faunes et flores exotiques...(2)

En conséquence, si l'on considère que les attitudes culturelles s'appliquent nécessairement aux clientèles potentielles du 
tourisme d'aventure, on ne peut qu'envisager une demande différente, propre à chaque pays - client. En somme l'Allemand, l'Américain, le Français, le Japonais... ont des attentes d'aventure très différentes et éloignées les unes des autres.

A titre d'exemple, nous dirons que l'Américain ayant déjà conquis le grand-espace, chez lui, et mis les pieds sur la Lune, ne considerera pas l'aventure comme un Français ayant colonisél'Afrique du Nord, ou un Anglais, qui aura étéinfluencé par les voyages de Darwin aux Galapagos.

Donc, si la demande est multiple, l'offreen tourisme d'aventure pour un même territoire devra l'être aussi; pas multiple en terme de beaucoup d'activités de plein air mais, surtout multiple dans le domaine des actes de vie Culture - Nature au sein du grand-espace: celui du Nord, des prairies, des déserts...

Ceci expliquerait - du moins en partie pourquoi au Québec nous ressentons une certaine difficulté à mettre en marché lo territoire amérindien de la Radissonnie, celui des Inuit de l'Ungava et de l'Hudson, ou plus simplement, celui de la BasseCôte-Nord. A l'inverse nous nous apercevons que le Polar berr en Ontario fait recettes - qu'en Alaska on recoit des centaines de milliers de visiteurs - et que le Wyoming, le Montana et les Dakota relancent leur tourisme d'aventure sur une base tres originale et compétitive. Il en est de même du petit paquebot, Rxplorer qui sillonne l'environnement aquatique en offrant lui, de l'aventure maritime.

En réalité, une de nos difficultés à redévelopper nos territoires d'aventure résiderait dans le fait que nous nous serions rendus prisonniers d'un modèle dominant

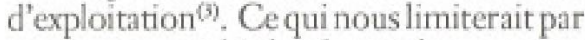
rapport aux multiples demandes couvrant un beaucoup plus grand éventail de clients. En clair, nous véhiculerions un certain tourisme d'aventure basé́ sur une nature âpre et rude... qui doit se mériter difficilement, au lieu d'être courtisée avec respect, facilité et sécurité( 4$)$.

Le tourisme d'aventure quel'on place très souvent dans le passé naturel se situerait plutôt dans un futur culturel recomposé au sein de grands espaces exceptionnels. Quant aux clientèles, celles-ci auraient aussi des attentes particulières reposant a priori sur leur

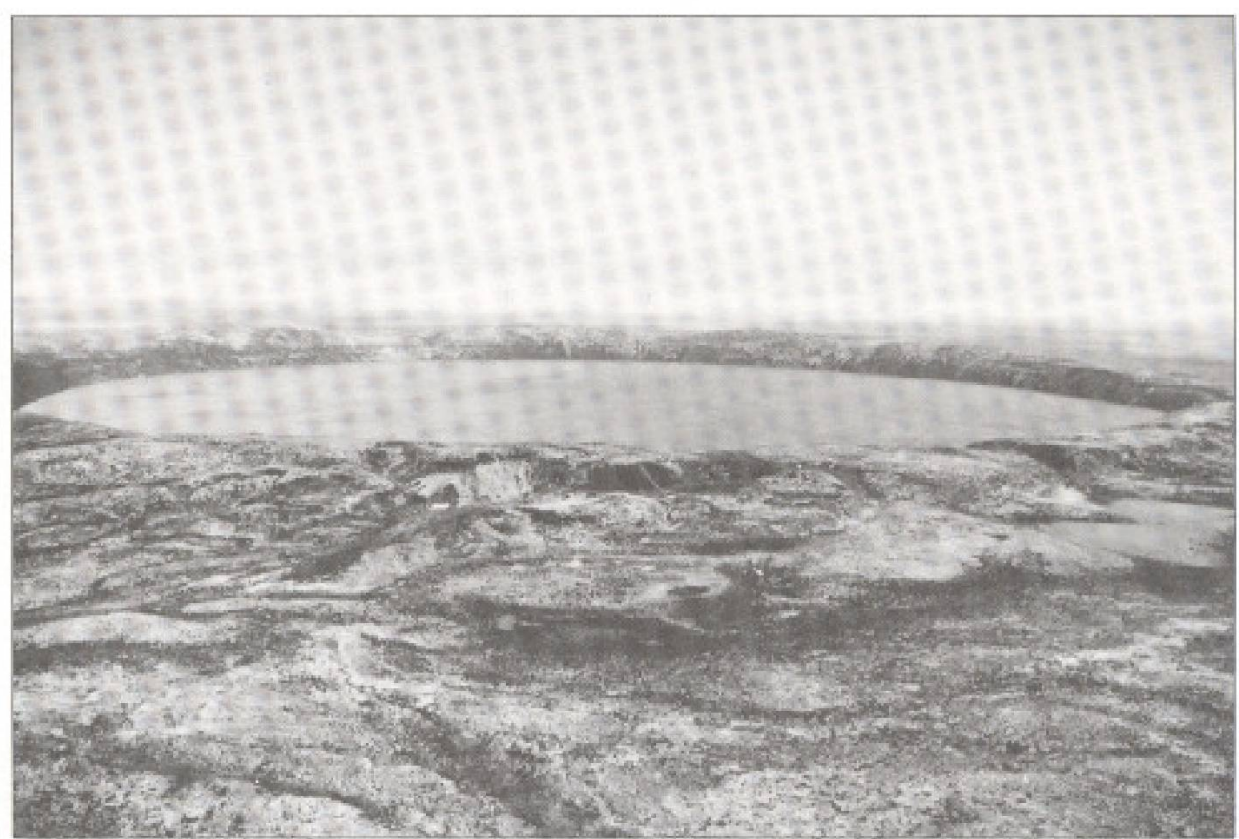

Le cratère. Le gatêre du Nunavik redkouvert par un Amóricain, en 1943 et son lac de crvatal, sont uniques au monde. De plus, a 90 kilometres environ, sur lile solitaire de Qikertaaluq, se trouvent les seuls peftroglyphes inuit connus, a quelques kilometres du village de Kangipsujuaq. Nouvelle synthese d construire entre les deologies et lart primitif. Photographe: Eugen Kedi

propre histoire, leur éducation et un imaginaire diversifié, dépendant du pays d'origine.

\section{Redéfinir le tourisme d'aventure... dans un cadre évolutif et prospect if}

Au Québec, c'est à Henri Jamet que nous devons une des premières définitions officiellesdu tourismed'aventure. Elledate de 1975, il y a presque vingt ans défà!

Il précisaità cette époque pas si lointaine, que le tourisme d'aventure avait débuté en 1973 avec une premiêre expédition dans le massif de Torngat. Toutefois, une revue de littérature approfondie et américaine, nous indique aussi qu'un tourisme d'aventure nord-américain existait au Québec dans la moitié du XIX ${ }^{\circ}$ siècle mais, qu'il portait sur des expéditions de chasseet pêche et de découverte de la culture amérindienneet Inuit ${ }^{(5)}$. Toujours selon le mëme document :

\section{L'Aventure avec un grand $A$ c'est d'abord qu'elle soit un engagenent plysique. Pour ale wows sacrifiez le confort, vous négligez la gastrono- mite, vous devenez un groupe altrwiste, gênérenx [...]. Vous wous}

adaptez à toutes les sitwations [...]. Les vacances aventures c'est decouvrir en profondeur d'autres terreset d'autres humains [... . C'est redécouvrir les émotions des pionwiers en taillant à la serpe son chemin dans wne foret vierge, en descendant des rapides en canot, en traversant des montagnes à dos d'étéphant, ou lé désert à dos de comeau [...l. C'est l'Evasion avec un grand $E$ [...] (Le towrisme d'aventure, p. 13).

Lavantage principal des définitions est qu'el les balisent une pensée sans pour autant l'enfermer dans un cercle définitif. Elles permettentaussi de comparer avecle temps, I'inflation des mots et des actes, ce que lion propose quelquefois aujourd'hui au nom d'une aventure, avec un petit a. Ce qui avantage sans doute quelques sites moyens deplein air mais, ce qui pourrait à la longue nuireà la notoriété du territoire du Québec. Car, le tourisme d'aventure ce n ${ }^{7}$ est pas n'importe quoi - n'importe où - pour n'importe qui - et pratiqué n'importecomment, derriere le village ou la ville la plus proche de Montréal ou Québed ${ }^{(0)}$.

C'est autre chose de qualitatif qui pourrait éventuellement se récrire de la façon suivante : 


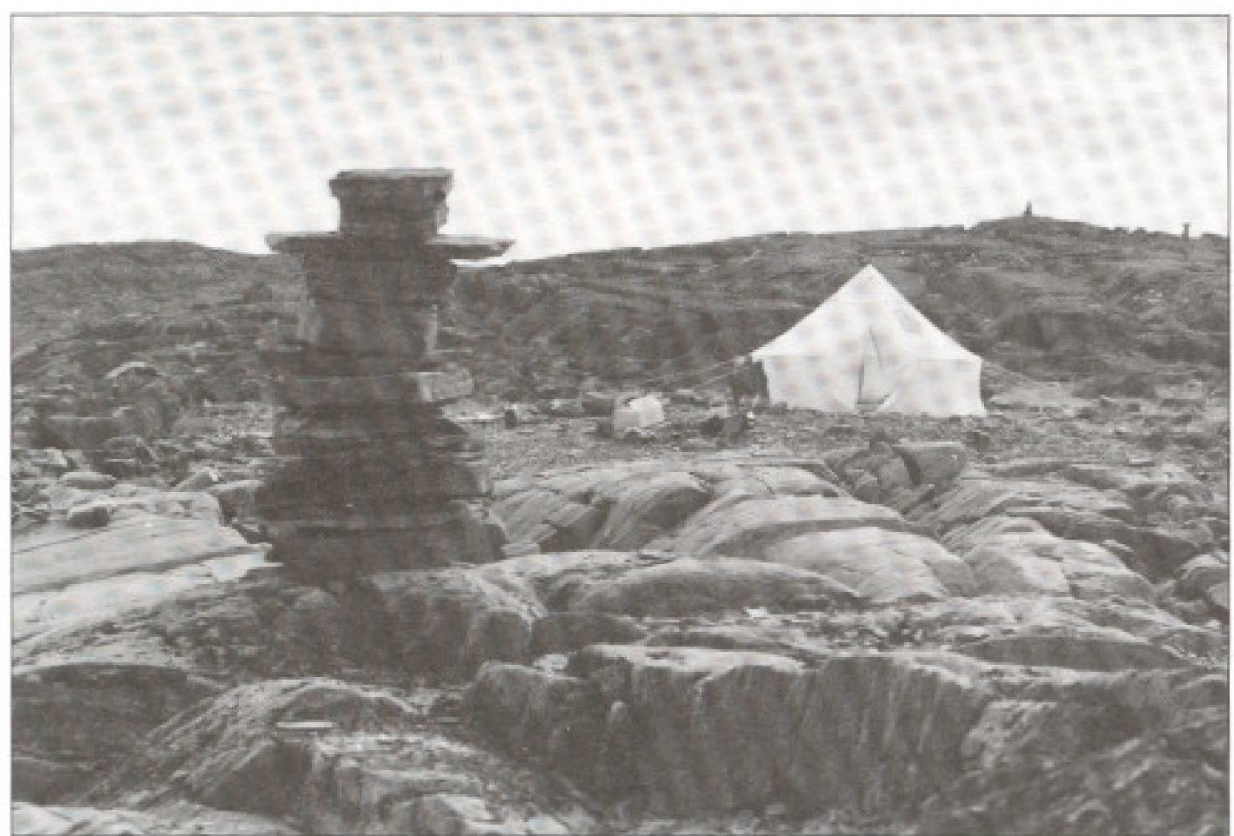

Les statues. Les Inukshuit, sorte de sculptures inuit ayant une utilité directionnelle, symbolique et magique, sont les seuls monuments nondiques. Dans un contexte futurogene, its devaient etre integres au redéveloppement du

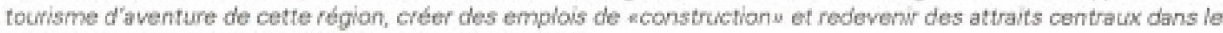
grandespace de la toundra. Photographe: Eugen Ked

L'Aventure avec un seul $\mathrm{A}$, est un acte de vie principalement culturel et temporairementvécu au sein des grands espaces naturels et évoca teurs quela terre a su conserver au regard de l'homme des années 2000. Pour s'y plonger, le touriste aventurier n'a plus besoin de sacrifier son confort, sa sécurité, ni de négliger son alimentation. Bien au contraire, les progrès techniques et scientifiques lui permettent déjà - à l'aube du troisième millénaire - d'expérimenter à un âgeavancé-des momentsintenses, dans des milieux isolés, là où jusqu'à présent, seuls des jeunes gens se risquaient à l'aventure.

De plus, le nouvel aventurier n'a plus à s'adapter aux situations dangereuses en puisant sa survie à même des environnements dorénavant protégés et devenus intouchables. Ses vêtements issus de recherches et fabriqués avec de nouveaux matériaux - sa nutrition équilibrée et diététique - ses moyens de locomotion relevant de la haute technologie - lui permettent d'accéder à tous les milieux et à les pénétrer comme jamais auparavant, pour mieux les connaître. Les communications par satellites lui assurent aussi un bien-être psychologique indéniable et un positionnement spatial sûr.

En fait, le tourisme d'aventure est devenu une Evasion plus contemplative... que

\section{Expériences d'aventures ou... activités de plein air communes?}

Alors que le Québec a amorcé - au sein du Canada et dans un contexte nord-américain - avec un certain décalage, le redé veloppement de son tourisme d'aventure, il semblequece dernier soit largement surreprésenté par des activités de plein air communes et des moyens de locomotion usuels. Le $4 \times 4$, le canoë, la motocyclette, le ski de randonnée, le kayak, le vélo de montagne, le véhicule tout terrain... sont beaucoup trop situés au premier plan de la mise en marché (L'aventure au Québec; $1500000 \mathrm{~km}^{2}{ }_{4 .+}$, p. 24).

Or, cette devanture, principalement axée sur la locomotion dans la grande nature n'est en fait qu'une simple activité outil qui devrait être assujettie de façon très secondaire à l'expérience de vie en situation de découverteetd'exploration. Un des exemples flagrants de la confusion entre l'activité de plein air extrênte et le tourișme d'aventure a été remarquablement exprimé sous la signature de Pierre Foglia, à la suite de sa... mésaventure au Tibet, organisée par le Club Aventure; lequel a dû s'excuser un peu plus tard.

\section{On voulait de la misere? On voulait du Moyen Age? On voulait de laventure nature? Des poux? Des gales? Un bol de riz pour deux? Messieurs les yuppies, vous êtes servis! Sauf que ce n'est pas un bol de riz pour deux qu'on a eu. C'est un bol de patates pour neuff. Le lendemain, l'estomac vide, nous attaquions le premier col [.... J. Vous me prenez pour un lama? Vous pensez que je levite? Au matin (du surlendenuain), le cuisinier tibétain nous servait des oeufs durs pourris. Et quand je dis pourris, je dis verdâtres. 7 e dis putrides, etc , etc. Gournal La Presse, 4 décenbre 1993).}

\section{Pour quelle aventure?}

En fait, si l'on resitue correctement le comportement humain universel au sein du milieu naturel sawtage - ce qui est souvent le cas de l'aventure - ce dernier n'est pas d'abord locomoteur mais plutôt perceptuel et cognitif(8), saufen cas de danger. 
En conséquence, le tourisme d'aventure est très éloigné d'une traversée pure et simple de l'espace ou du désert... au sens biblique, et son intếrêt réside essentiellement à l'intérieur d'une façon devivre temporaire et isolée au centre d'une immensité grandiose et esthétique, à contempler. A cet effet, le sentiment de vastitudeetde découverte est plus périphérique et panoramique que linéaire. Et ceci concerne tout l'aménagement et le développement du tourisme d'aventure peu importeoù on lesitueau Québecetailleurs.

De façon concrète, nous devons alors reconnaütrequeles grands raidsen Amazonie, en Afrique (Paris-Dakar), au Québec (Harricana)... sont avant tout des proteesses médiatiques et sportives. Elles n'entraînent à peu près rien comme développement économique durable\%", ni pour les autochtones, ni pour les résidents des localités traversées... à grande vitesse!

Que pourraient alors être les éléments de solution dans un cadre prospectif allant jusqu'en 2010 enviton? A notre avis, il s"agirait de corriger la démarche actuelle en ne véhiculant plus de simples images et activités de plein air qui ont connu un certain succiss aux alentours des années 1960-1980. Il faudrait plutôt constrmire des expériences de vie environnementales à caractère original, adaptées précisément aux grands espaces concernés. D'autre part, il faudrait éviter de trop mettre en marché un espace informel et relativement phobique - le $1500000 \mathrm{~km}^{2}$ du partout et du nulle part = et se concentrer dorénavant sur quelques lieux concrets et significatifs à remettre en valeur.

Cette tendance à la précision et à la concentration autour de gisements environnementaux, concerned ailleurs non seulement le secteur aventure du tourisme- mais aussi - toute l'industrie touristique en général. On se déplace de plus en plus pour l'ambiance d'un certain quartier à Londres, New York, Paris... - ou encote - pour certains sites bien circonscrits du Nord canadien, des prairies de l'Ouest, des désertsen Arizona, au Colorado... Quels sont exactement ceux de la Radissonnie, de l’Ungava, du Témiscamingue et du Nord en général ?

Le tourismed'aventure semble deplus en plus se constituer autour d'expériences complètes vécues pendant une à trois semaines environ ${ }^{(11)}$, Ceci replace l'activité de plein air locomotrice dans un contexte plus culturel que primaire du type: activité physique dans la nature! Quant aux lieuxd'aventure, ceux-cidevraientêtre atteints en un laps de temps minimum pour, ensuite, etre vécus... plus longuement. Il s'agit donc de les sélectionner avec rigueur dans le grandespace, a priori, indéfini. Quant à son aménagement, celui-ci devraits' effectuer en coquille plutôt qu'en circuit linéaire trop éprouvant et trop long.

\section{Le tourisme d'aventure... une rare culture dans une immense nature}

Cela peut paraitre surprenant mais, un des principaux motifs de l'Aventure humaine en référence à l'Écologie humaine - serait de redécouvrir deséléments culturels rares etprécieux au sein de la grande nature. Qu'il sagisse d'ecosystemes uniques, de paysages grandioses, de cités enfouies ou de modes de vie conservés par l'isolement...

L'Amazoniestintéressante, surtoutà cause dela protection du mode devie amérindien et des écosystèmes forestiers; ce qui s'apparente directement à l'Écoethnologie, disciplines scientifiques. L'Australie est surtout attirante en rapport avec l'imaginaire des Aborigènes et un art rupestre particulièrement somptueux au sein des grands espaces semi-désertiques - et ce dans un rapport ethnoartistique. Les Amérindiens des plaines commencent à fasciner les touristes aventuriers par l'architecture du tepee, la redécouverte de centaines de médectine whels ${ }^{(1)}$, d'iniskin ou piorrer de bison, qui remontent à 5000 ans. Quant aux Inuit du Québec et du Canada, ceux-ci sont connus dans le monde entier, surtoutau travers del'architecture del'igloo, de leurs sculptures symboliques, de l'érection de Inukshuit ${ }^{(12)}$, des masques gravessur l'île Kuikuatarluq, dans le détroit d'Hudson..,

En réalité, il nous faut reconnaître qué l'intérêt majeur de l'exploration moderne est surtout une expérience culturelle exceptionnelle, et non, une prouesse physiologique ou biologique dans un environnement hostile; une sorte de terre de Cäm. En conséquence, il serait donc utile detraduire la demande implicite du touriste aventurier par une approche axée sur les cultures prinutives, et les richesses naturel- les, contenues dans les écosystèmes particuliers.

Ledilemmeesttel qu'actuellement, le point de jonction de la pratique du tourisme d'aventure se situe fréquemment dans une nature rendue volontairement extrême par un certain type de programmation, et une rencontre des populations locales, qui refusent de se présenter sous un aspect folklorigue.

Ce genre de situation est surtout vécue par les Inuit et les Amérindiens à qui l'on conseille à grands frais, et sans trop de résultats socio-économiques, un tourisme d'aventure... par le kayak de mer, la motoneige, le traîneau à chien, la chasse et la pêche... Autant d'activités remarquablement organisées par le Sud, qui retiennent déjà l'ensemble des clientẻles rentables, avant qu'elles atteignentles territoires du Nord, et le $50^{\circ}$ parallèle!

La liste de ce genre de malentendus, rattachés d'une part, à une forme de déplacement de la pratique de l'aventure sur des sites conmuns; à laquelle s'ajoutent d'autre part, des phénomènes d'acculturation et d'evolution des modes de vie ancestraux, est très longue au Québec, et partout au monde.

Mais alors, où découvrir des éléments de solution de façon à mieux satisfaire les parties en cause? Nous dirons, dans le présent des environnements et des cultures locales mais, en ne conservant et en n'adaptant que l'essentiel et le meilleur du passé! Qu'ils'agisse du langage, des rituels fondamentaux, de l'architecture, des événements principaux, de l'art.. En somme, il s'agirait d'être capable d'agir vers le futur comme on le fait déjà remarquablement vers le passé, au travers des living museum; ces portionsd'espace-temps soigneusement protégées ou reconstituées ou encore... conservées.

Cette approchen'est pas la plus facile mais, elle est assurément une des plus prometteuses. Et elle est relativement simple à composer, pour peu qu'on la situe en dehors des parodies du passé et des productions de pacotille pour le futur. Quelques exemples sont déjà en place, qui démontrent bien quele tourisme d'aventure - du moins en Amérique du Nord - est en mutation, comme en Australie ${ }^{(13)}$ ou en Afrique du Sud. 
Par exemple, dans le Montana, au sein des immenses plaines herbeuses de l'Ouest, proche de Cheyenne River, là où les Sioux se rểunissaient il y a de cela un siècle, à quelques kilomètres du site sacréde Mateo tepeeet du lieu de défaitedu général Custer en 1876, a été reconstitué un hébergement touristique sous des tepee modernisés. Certains pourraient arguer qu'il ne s'agit pas là d'un vrai tourisne d'aventure en soi, et quel'authenticité et l'exotisme font défaut! Erreur dirons-nous car, dans ce cas précis, et dans bien d'autres aussi, il s'agit d'une authentique montagne sacrée, mondialement connue ${ }^{(14)}$, d'un non moins véritable grand-espacemythique-d"authentiquersites évociteurs remontant à la civilisation du bison, de vrais tepee confortables, de vrais événements historiques...

Sans pour autant copier à la lettre un tel modèle mais, en ne retenant que les fondements de ces produits de la nouvelle génération aventure, nous devrions aisément proposer des innovations semblables et équivalentes sur le marché international, à partir du Québec.

Par exemple, le cratère du Nouveau-Québec et sa région seraient à privilégier sur le plan du mythe, de la science, du paysage et de la culture Inuit. Il en serait de même du village amérindien moderne - traditionnel d'Ougé - Bougoumou placé lui, à la porte dela taigga, à proximité rela tived'unegrotte sacrée... Quant à la Basse-Côte-Nord, il s'agirait simplement d'accélérer le développement des trois archipels d'Harrington Harbour, de Sainte-Marie et de Providence, pour faire surgir un tourisme d'aventure maritume de grande qualité ${ }^{\prime 15}$. Car c'est là que le peintre animalier Audubon s'est inspiré d'une faune unique, que la technique de pêche du loup-marin a été inventée, et que l'histoire s'est concentrée un moment.

Le tourisme d'aventure de nouvelle génération est en émergence sur les cinq continents, et le Québec possède des sites exceptionnels à mettre en valeur en collaboration étroite avecles cultures Inuit, Amérindienne et... Nord-côtière, en particulier. La principale difficulté pour constituer ces produits originaux de calibre international, est de savoir les bâtir en concevant une nouvelle synthèse, à des endroits précis du territoire. Une synthèse essentiellement culturelle, capable de traduire le grand-espace par le mythe, la religion, l'histoire, la science, l'art...

\section{Conclusion}

Comme on le constate de plus en plus, le tourisme d'aventure, souvent relié à l'écotourisme et au tourisme vert, capte un nombre restreint de clients et engendre une rentabilité qui ne demande quara augmenter mais, sur des bases plus culturelles et perceptuelles que naturelles et physiques. Ce qui, en conséquence, devrait amener l'ensemble des organisateurs de cette branche touristique, à parfaire leurs connaissances dans plusieurs domaines jusqu'ici négligés... ou ignorés.

De plus, le tourisme d'aventure basé sur l'observation des dernières cultures primitives immobilisée dans le temps, et des écosystèmes figtés, connaîtra dans un avenir prochain un certain plafonnement. Et cela, parce qu'il repose trop exclusivement sur un passé en mutation rapide et... non reconductible. Les modesdevieancestraux résiduels, ainsi queles écosystèmesévoluent dorénavant de façon accélérée et irréversible. Ils se seront à peu près tous transformés dans les dix prochaines années.

Les nouvelles aventures ont donc tendance à se tourner vers un imaginaire accru, au sein d'environnements sensoriels et cognitifs exceptionnels, où le mythe et l'image syn thèse domineront l'expérience vécue. En quelques heures, en quelques jours, on souhaitera de plus en plus atterrir ou amerrir quelque part dans le grandespace, et comprendre très rapidement ce que des milliers d'années ont produit avant nous, en terme de Culture - Nature. Les futures aventuresrelèveront ainsi beaucoup plus de la responsabilité de gens cultivés que... simplement performant au niveau musculaire! Il faudra aussi être capable d'agir vers le futur en priorité, plutôt que d'effectuer de constantes et trop nombreuses regrenions passéistes.

Pour reconstituer les produits aventures, il s'agira alors, à partir de territoires soigneusement calibrés, de bâtir des expériences de vie intenses et trèे riches en connaissances syntbétivécs. Etcettenouvelle offre, plus conforme à la demande, ramènera l'activité de plein air au rang d'accessoire utile, sans plus! Elle modifiera aussi l'offre en hébergement, restauration, dé- placement, séjour, relation avec les gens et le milieu physique.

Quoique certaines recherches indiquent que le tourisme d'aventure pourrait progresser de $25 \%$ à $30 \%$ dans les années à venir, il appartient à un marché restreint de hautequalité, qui resteà parfaireou même... à refaire!

En général, le touriste aventurier est un voyageur bypermotrvé qui pratique un tourisme, frugal et simple qui coutte cher. En conséquence, il exige que ses découvertes sur le terrain, en terme de milieux et de services, soient à la hauteur des images qui l'ont attiré à un endroit prédéterminé.

Or, il faut en convenir, il y a souvent un grand écart entre la publicité et la potentialité remarquable du territoire québécois, et la traduction concrète que l'on en fait. C'est ce décalage qu'il s'agirait entre autres de réduire au minimum, de façon à ne pas perdre la part du marché international qui nous revient.

Enfin, le tourisme d'aventure de nouvelle génération concerne directement l'ćconomie de plusieurs régions périphériques du Québec, et situe le développement audelà des activités de chasse et pêche traditionnelles. Nous entendons par là que les Amérindiens et les Inuit en particulier, pourraient avoir plus intérêttà redévelopper leur culture sous des aspects originaux, au sein de leurs nouveaux territoires, qua persister dans des champs déjà occupés par la concurrence: pourvoiries, bases de pleinair, centres d'expéditions... Nous avançons même l'idée que les Autochtones - excellents guides et gestionnaires de certaines pourvoiries - auraient avantage à bâtir un tourisme d'aventure pour les années 2000 , autour de la tradition de subsistance touchant la chasse, la pêche, la cueillette, leur histoire et leur mythe... Cette approche convenant beaucoup mieuxà leur culture, que la simple gestion des territoires fauniques dans laquelle on essaie de les introduire, pour d'autres fins ${ }^{(16)}$.

\section{NOTES ET REFFERENCES}

(1) Qui défie le temps... ou qui regroupe toutes les actions du temps dans une sorte de synthese.

(2) Ce qui est principalement a l'origine des activitós de plein air et place l'activité physique au second ou troisième rang. bien après la connaissance directe du mileu et la perception esthétique.

[3] Copie sur la France et la Nouvelle-Zelande en particulier, sans grande adaptation. 
(4) Club Aventure: Tibst [...] LeClub Aventuren'a rien négligé, bienaucontraire l... Jila retenu les services dun cycliste aguerri... professeur |... | de plein air de Tróis-Rivieres... 18 décembre 1993. Aguerrir: habituer aux dangers de la cuerre.u Analogie significative entre le tourisme d'aventure, le plein air, le combat. .. au lieu de l'harmonie et du plaisir!

(5) Ce qui a aide a la propager au niveau international.

(6) La vulgarisation est telle qu'elle tend parfois... a la parodie aventureuse, banale, commune...

(7) Plus intellectuelle et ecologiquement humaine aussi!

(8) Ce double comportement ira en augmentant ot suiva la courbe ascendante des connaissanceset communications à I'aube des annése 2000-2010.

(9) En somme cela est pou utile au développement économique local et régional et ne génère aucun emploi stable de qualité.

10) Plus exactement, a partif de trois jours, pour une excursion de plein air jusqu'à trois semaines pour une expedition aventure, pour une moyenne globale de huit jours

(11) Roues spirituelles datant d'avant Stonehenge et d'avant les pyramides d'Égypte; c'est-ä-dire audelà de 5000 ans.

(12) Statues faites de pierres empilees qui ont une valeur magique et une utilité diféctionnelle dans la toundra.

(13) Dans le mème ordre d'idées, l'Australie vient de faire peindre un boeing 747-400 en sinspirant du netreart aborigene. Cet avion ultra-fnoderne portera dans le mande entier les symboles graphiques issus directement du mode de vie primitit. lui-meme extrait du grandespace australien. Exemple de jonction concrète entre le passé et le futur.

(14) Entre autre, par l'intermédiaire dufilm Rencontre du $3^{*}$ type. Mateo tepee: la montagne griffée par un ours géant!

(15) Ce projet de tourisme d'aventure maritime remonte déa a quatre ans et a comme objectif economique de compenser les pertes encourues par la pénurie de poissons dans cette zone.

(16) Les Amérindiens et les Inuit dirigeant avec brio des pourvoiries de renom comme Le Vieux-Poste sur la lac Mistassini - Fingers Lake di Riviere-auxfeuilles - Tuktu sur la rivière George... Mais est-ce wraiment les formes de chasse et póche reliées à leurs cultures ancestrales et celles qui conviennent le mieux au tourisme d'aventure? Nous n'en sommes nullement convaincus!

\section{REFFERENES}

ADNENTURE THAVEL ASSESSMENT REPORT, The Lannon Group Inc., Overseas Adventure Travel. Canadian Consultate General ${ }_{\text {,Baston, The Quebec }}$ Government Delegation for New England, Air Canada, Air Alliance, 1990

ADVENTURE TRAVEL IN BRITISH COLUMBIA, Summary of Findings, The Outdoor Recreation Council of British Colombia, Ministry of Econorric Development, Tourism Recreation and Culture, 1988

ADVENTURE TRAVEL INA EASTERN CANADA, AN Ovarview of Product and Market Potential, wol. 1: Final Report, Tourism Fesearch Group, Ethos Consulting Ltd., Tourism Canada, 1990

ALLARD, Lionel, Tibet: Club Aventure plaide coupable avec explications, Journal La Prasse, 18 decembre 1993.

ASSOCIATION NATIONALE POUR LE DEVELOPPEMENT LOCAL ET LES PAYS (ANDLP) ET L'INSTITUT DE FORMATION EN DEVELLOPPEMENT ECONOMIQUE COMMUNAUTAIRE (IFDEC), LE local en action, Editions de l'Épargne, 1989

BARAĖ, D. et A. LEFEBVRE, Étude sur l'inventaire des ressources du milieu biophysique et du potentisl rúcréatif de la région Korec-Torngat, au Nouveau-Québec, MLCP, 1986.
BENKO, Georges et Alain LIPIETY, Les régions qui gagnent: districts ot réseaux: los nouveaux paradigmes de la géographie Éeonomique. ouvrage collectif, Collection Economie en liberte, PUF, 1992

BOUCHARD, Michell et al. L'histoire naturelle du cratère du Nouveau-Oúbec, 1989

CETRON, M.J and W. ROCHA, Travel Tomorow: The Haspitable Future, Futurist, vol, 21, no 4, 1997.

CHISASIBI TOURISM DEVELOPMENT, sotar and Chisas ibl Tourist Agency. 1989

ETUDE DE DEVELOPPEMENT DE LA BAIEJAMES. vol. 2: Options do dóvaloppement bconomique, Sores inc. et Battelle Memorial Institute, S.D.B.J., 1973

Fógla, Plerre, Foglig au Tibet: un bout d'enfer, Journal La Presse, 4 décembre 1990.

GAUTHIER, Claude, Le développement du tour isme d’aventure dans le Nord qub́bbcois. Le cas de Shefferville, mémoire de maitrise, Université de Sherbrooke, 1966

GERMAN, Georges-Hébert, Mon anark, ma brosse a dents. Le Grand Nord quebecois est lune des dernieres regians vierges du giabe, fitime destination du fourismed'aventure, L'Actualitá, 1987.

GOUVERNEMENT DU QUËBEC, $1500000 \mathrm{~km}^{2}$ d'aventure, 1990.

GOUVERNEMENT DU QUEEEEC, Tourisme Qứbec, Les produits touristiques et lours retombees bconomiques, produit aventure, $1994, \mathrm{PP} .60$ 62.

NDUSTRIE, SCIENCE ETTECHNOLOGIE CANADA, Le potentiel de dévoloppement touristique de la Basse Cate-Nord, 1991

INDUSTRIE, SCIENCES ET TECHNOLOGIE CANADA Recherche sur le dóvaloppement et la commercialisation du produit tourisme d'aventure du Québec, recherche interme, 1991.

INDUSTAY, SCIENCE AND TECHNOLOGY CANADA, Federal Tourism Strategr - Northwest Torritories. 1990.

INSTITUT DE FORMATION AUTOCHTONE DU QUÉBEC, Actes du colloque sur le dóveloppement du tourisme en territoire autochtone, tenu à Chicoutimi, 1991.

ISOGROUP. Plan de développement touristique; rógion Nord du Ouábee. 1993.

JAMET, Henri, Le tourisme d'aventure, ministère du Tourisme, Quebec, 1985.

JAY-RAYON, Jean-Claude et collaborateurs, PCL de Harrington Harbour - Sainte-Marie at Providence; Sea Shore Adventure Tourism, portefolio et documents annexes, 1991.

JAY-RAYON, Jean-Claude, encollaboration aveo Brigitte Morneay, Prospective touristique regionale ef iocale, 1995-2010: scénarios tendanciels et explaratories, Teor os, no 2, juillet 1993.

JAY-ABYON, Jean-Claude, en collaboration wec Luo Trepanier (cartographie), Basse-Côte-Nord: Lacolte des anchipeis; win projet récré-touristique novaaur dans les lies Harington. Providence et SainteMane, Rovue Continuité, no 52, 1992, pp, 42-47.

JAY-RAYON, Jean-Claude La valour économique du patrimoine - envir onnement, adaptation de la démarche de Xavier Greffe etcollaborateurs, 1994.

AY-RAYON Jean-Claude, Le rácrétourisme et Is planification stratégiqueréglonale, ministére du Conseil exécutif, Secrétariat aux Affaires regional. les, 1994

NAVARFO, G. CUMPERS, C, et B. MATHIEU, Harricana Qudbec Canada, Les Editions Solar, 1990.

PEAT MARWICK ET ASSOCIESS; CAEE REGIONAL AUTHORITY, Cree Tourism Development Plan: A Discussion Paper, 1979.

PEAT, MARWICK ET ASSOCIÉS, CREE REGIONAL AUTHORITY, Preliminary Tourism Analysis. Cree Community Visits, 1979

TANGRAM, Affiche et prospectus du Sea Shove Resort Adventwe de Harrington. Sainte-Marie et Providence, 1 affiche, 1 prospectus, 1994.
Tepee Tranquility. A New Generation of Westemers, Travel and Loisure, June 1994

THE NUNAVIK TOURISM NEWSLETTEF, Lac du Cratère du Nouveau-Qubbec, pr thistoire, histoir e et ar cheologie dans la région Kangiqujujua et de Pingualuit, Makivik Corporation, Special Edition, 1994.

THE OPEN POLYTECHNIC OF NEW ZEALAND, Developing the Adventure Tourism Professional, An Adventure Tourism Training Opportunity. 1992

TOURISM AESEARCH GROUP, ETHOS CONSULTING LTD., TOURISME CANADA. Tourisme d'aventure dans l'Est du Canada - Apercu du potentiel des produits et marchós, vol، 1: Rapport deffinitif, 1950.

TOURISM RESEARCH GROUP. ETHOS CONSULTINC LTD., TOUAISME CANADA, Tourisme d'aventure dans l'Est du Canada - Apercu dupotentie desproduits ot marchess, vol. 2: Annexes, 1990

TOURISME CANADA, Évaluation des voyages d'agrément des Allemands, 1969.

TOURISME CANADA. Evaluation des voyages d'agrément des Baitanniques, 1908.

TOURISME CANADA, Evaluation des voyages d'agrément des Français, 1989

TOURISME CANAOA, Evaluation des voyages: d'agrément des Japonais, 1988

TOURISME QUÉBEC, TOURISME CANADA, Québec Fishing \& Hunting Packages, 1990. 